Proceedings

\title{
Study of the Architecture of a Smart City ${ }^{+}$
}

\author{
Jose Antonio Rodriguez ${ }^{1, *}$, Francisco Javier Fernandez ${ }^{2}$ and Pablo Arboleya ${ }^{2}$ \\ 1 Gijon City Council, Plaza Mayor No. 3, 33201 Gijon, Spain \\ 2 Polytechnic School of Engineering, Oviedo University, 33203 Gijon, Spain; javierfernandez@uniovi.es \\ (F.J.F.); arboleyapablo@uniovi.es (P.A.) \\ * Correspondence: jarcortes@gijon.es; Tel.: +34-649-189-290 \\ + Presented at the 2nd International Research Conference on Sustainable Energy, Engineering, Materials and \\ Environment (IRCSEEME), Mieres, Spain, 25-27 July 2018
}

Published: 12 November 2018

\begin{abstract}
In recent years, we are experiencing a digital revolution in many contexts, the digitalization of industry (Industry 4.0), the digitalization of public administrations, the digitalization of people and things (Internet of Things) and, of course, the digitization of cities under the name of Smart Cities. Although the digitization process is based on 3 main pillars: data, talent and innovation. It is necessary to make a mixture of these 3 pillars in different percentages to achieve digitization. In the case of cities, great weight lies in the technology deployed that makes up the architecture of the city. This technology is very incipient and lacks standards that allow interoperability between different devices. This article tries to show a picture of the possible architectures existing today from the point of view of sustainability and energy efficiency.
\end{abstract}

Keywords: smart city; internet of things; sustainability; efficiency; energy

\section{Introduction}

Why should a city transform itself into a Smart City and start managing its resources efficiently? According to the United Nations [1], currently more than $54 \%$ of the world population lives in a city, the forecast for 2050 is to reach $66 \%$. This growth will be a huge challenge for the infrastructure, resources both.

To overcome this challenge, it will be necessary to analyze the data generated by the sensor network, apply optimization algorithms using artificial intelligence techniques or neural networks, which on the one hand are capable of analyzing a huge amount of information from multiple sources, and on the other hand, be quick enough to make decisions.

\section{Smart City Definition}

In recent years, Smart City term has become fashionable and sounds increasingly in technological forums, political institutions and even in the day to day of citizens. However, really, do we understand the scope that this term describes? In order to understand the scope, it is necessary to establish a definition that encompasses the entire scope it covers.

If we look in the literature there is no single definition, according to the White Paper of Smart Cities [2], the purpose of a Smart City is to achieve efficient management in all areas of the city while satisfying their needs and the one of its citizens. At the same time, it must be aligned with the principles of Sustainable Development and taking technological innovation and cooperation between economic and social agents as the main drivers of change.

According to Spanish Association for Quality, a Smart City [3] is one that uses technological advances as a support and tool to improve the quality of life of inhabitants. In other words, these cities are in which investments in human and social capital and communication infrastructures 
promote sustainable development and a high quality of life, with adequate management of resources through participatory governance.

As you can see, certain common aspects are identified (technology, citizens, improvement of the quality of life) but there are terms that are not common among them (participative government, human capital, etc.), besides, the form of perform an efficient and sustainable management of resources or even more, which are sustainable resources or efficient management of them.

\section{Architecture of a Smart City}

After analyzing multiple models applied in different cities to fit within the definition of Smart City, what is appreciated is the intensive use of technologies and especially telecommunications technologies to achieve resource monitoring. It is here, where you can identify a mechanism for the efficient management of resources, the need to monitor energy resources, humans, etc. To, in some way, manage them in a more efficient way.

Then, we can introduce the term "monitoring" within the definition of Smart City to establish a mechanism that allows us to manage efficiently and sustainably. In order to monitor a city, we must identify the different zones or parts that compose it and that each one will have its specific casuistry. It is not the same to monitor the flow of vehicles that circulate in the city center that the flow of people circulating inside a train or bus station. The type of sensors, communication protocols, data analytics, etc. will be different.

In a Smart City, you can identify two types of architectures, the one that monitors the exteriors of the city such as its streets and avenues, parks and leisure areas, etc. and the one that monitors the interior of buildings, the flow of people and goods, air conditioning, water, etc.

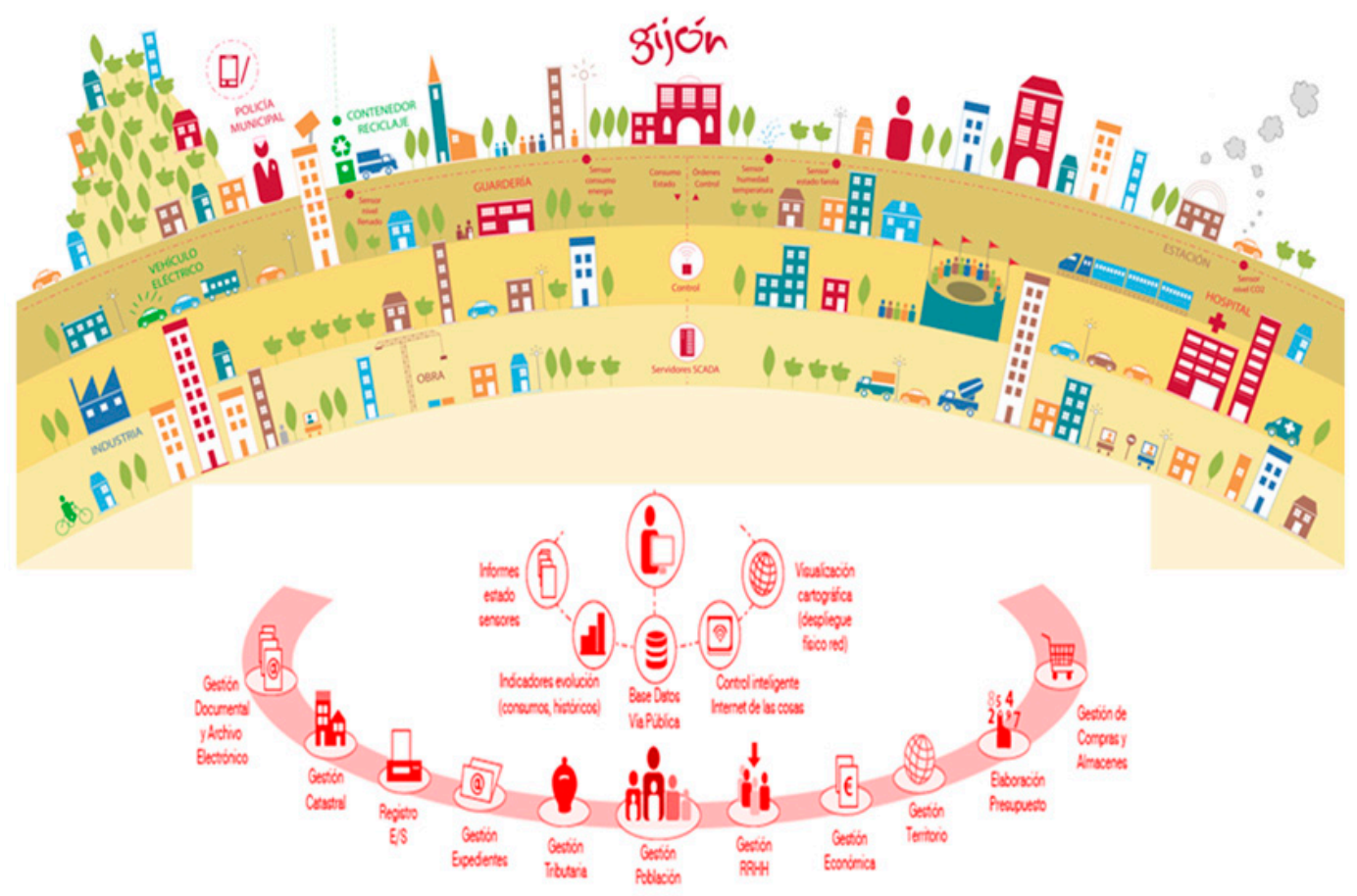

Figure 1. Gijon Smart City model (Image courtesy of Gijon City Council).

The architecture deployed in a city for external monitoring is based on long-range communication protocols, so that the entire city can be covered with few devices. These communication protocols cannot penetrate into buildings due to interference with walls and electrical or piping installations.

The architecture deployed in buildings usually take advantage of the telecommunications infrastructure itself or, failing that, the wired electrical network, it is easier and cheaper to rely on these facilities. In areas of difficult access or without wired installations, wireless protocols such as 
Wi-Fi, Bluetooth or RFID are used. These protocols are short-range and have coverage from $0.5 \mathrm{~m}$ to $10 / 15 \mathrm{~m}$.

A correct selection of external and internal architectures adapted to the geometries and distances to be covered will allow a correct communication with all the sensors and equipment of the city and a correct and without errors or latencies of the necessary data for the analysis and optimization of resources.

\section{External Architecture}

To know what happens in a city, it is necessary to place a series of sensors that allow obtaining certain parameters and variables for making decisions about the processes that occur in the whole environment. On the other hand, there are many sensors that provide different architectures to the city, the most common are the star architectures or mesh network.

\subsection{Placement of Sensors}

Most cities that are implementing a smart city model have opted to place sensors on public light, what is the reason for this decision? This responds to several reasons:

- Public lighting is an essential city system, so it is an element present in all urbanized cities.

- It is a wired network, so electric power is available to power the sensors.

- The elements can be placed at a certain height, avoiding vandal acts.

- The arrangement of the luminaries responds to lighting needs of the street in which they are placed, so there will never be a lack of places to place the sensors.

- If the sensors monitor the luminaries, we will have a system for remote management of the lighting, so that energy consumption can be adjusted and optimized.

There are cities where it has been chosen to place the sensors in other elements of the city such as garbage containers, litter bins, bus shelters and even in certain strategic points of the city with an independent placement of any element of the city. All possible locations have greater disadvantages than the option of placement in public lighting.

\subsection{Infraestruture}

The different technologies for the sensors of a smart city generate an infrastructure on the city, the most common models are the infrastructure in star or mesh network.

Star infrastructure makes the sensors scattered by an area connect directly to an access point that sends the information to the database for collection and further processing.

Meshed network infrastructure allows the sensors to connect to each other and through the sensors closest to the access point; the information is dumped into the database.

\section{Internal Architecture}

The way to approach the placement of sensors inside a building has a great dependence on the following parameters:

- The geometric shape of the building itself.

- If the building is considered a heritage asset or not.

- The use of the building, it will not be the same to sensor an office building as a sports hall or a train station.

- Ownership of the property, public or private.

- New or existing building

In new buildings, the architecture and sensor layout is another milestone in the planning. However, in an existing building, it is necessary to evaluate the parameters set out above and establishes a correct deployment of sensors and actuators for the proper functioning of the IoT system. 


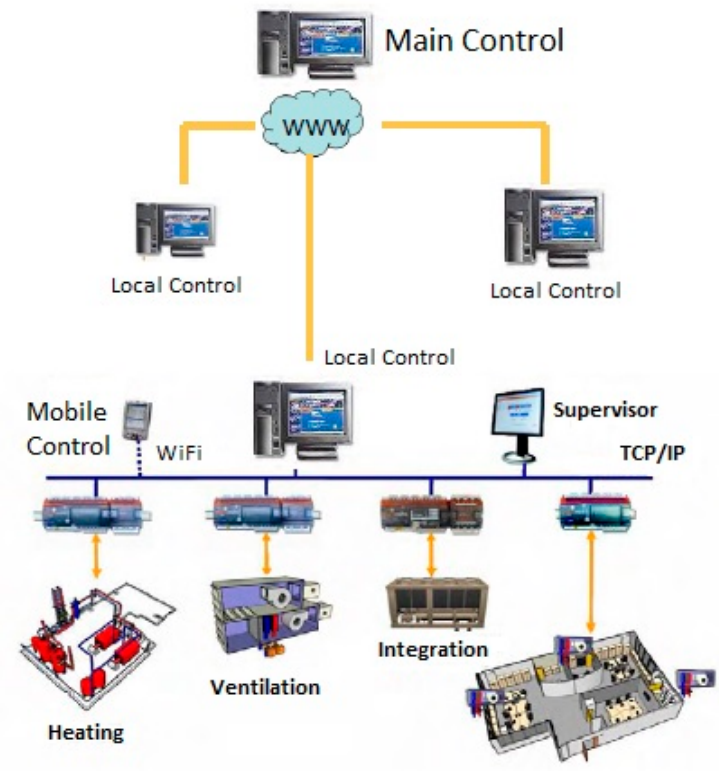

Figure 2. Schema of Smart Building sensors (Image courtesy of Gijon City Council).

\section{Sustainability and Energy}

One of the strengths of IoT is its direct application for measures of sustainability and energy efficiency. Sensor infrastructure deployed in a city allows knowing how, where and when the energy is being consumed. All this information allows generating different consumption profiles for the city objects (lighting, transport and buildings) and through this knowledge, to optimize consumption avoiding waste.

Lighting: by means of sensorización it is possible to include presence detectors and reduce the luminosity of the lighting when there is no presence of pedestrians. In addition, the introduction of LED technologies, easier to regulate digitally, allows savings of up to $85 \%$ in electricity consumption.

Transport: people counting sensors allow optimizing urban transport fleets reducing the consumption of fossil fuels. Control position allows reducing grouping-effect of lines and improving access to the citizen.

Buildings: the integration of the information of all the energy consuming elements of a building (HVAC, water, light, access, fire, security, etc.) allows to optimize the load profiles against their demand, drastically reducing unnecessary consumption. It is also possible to control energy consumption due to oversights such as leaving lights on or heating, allowing remote shutdown.

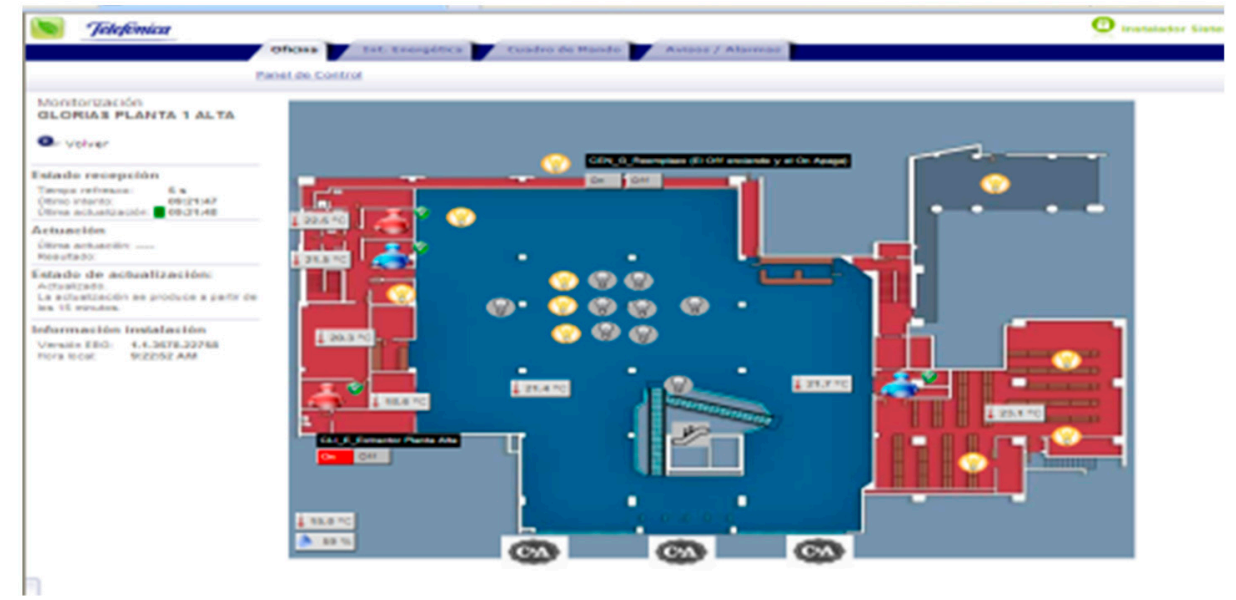

Figure 3. Web services light management (Image courtesy of Telefonica). 


\section{Conclusions}

The disruption of IoT and new communication technologies will allow, in coming years, a drastic reduction of energy consumption globally and with it, our environmental impact and carbon footprint.

For cities, it will be a giant step towards sustainability and resources optimization for super-population problem that is predicted in about 30 years.

We are at the doors of a new digital era where the sensorial will be an integral part of our lives.

Acknowledgments: Gijon City Council for its permission for the realization of this article.

Conflicts of Interest: The authors declare no conflict of interest.

\section{References}

1. United Nations. Available online: http://www.un.org/en/development/desa/news/population/world-urb anization-prospects-2014.html (accessed on 2 May 2018).

2. Telefonica S.A. Available online: https://iot.telefonica.com/libroblanco-smart-cities (accessed on 1 May 2018).

3. Asociación Española para la Calidad AEC. Available online: https://www.aec.es/c/document library/ get file?uuid=c8b4e9a6-1796-4e90-9ddd-4ceb3ac66a81\&groupId=10128 (accessed on 5 May 2018). 